\title{
Le panda hypocrite : sinité, studios d'animation et discours orientaliste postmoderne
}

\section{Pierre-Mong LIM}

\section{(2) OpenEdition}

\section{Journals}

Édition électronique

URL : http://journals.openedition.org/transtexts/408

DOI : $10.4000 /$ transtexts.408

ISSN : 2105-2549

Éditeur

Gregory B. Lee

Édition imprimée

Date de publication : 7 avril 2011

ISSN : 1771-2084

Référence électronique

Pierre-Mong LIM, «Le panda hypocrite : sinité, studios d'animation et discours orientaliste postmoderne », Transtext(e)s Transcultures 跨文本跨文化 [En ligne], 6 | 2011, mis en ligne le 07 avril 2011, consulté le 20 avril 2019. URL : http://journals.openedition.org/transtexts/408 ; DOI : 10.4000/ transtexts.408 


\title{
Transtext(e)s Transcultures 跨文本跨 文化
}

Journal of Global Cultural Studies

6 | 2011:

Debating China

\section{Le panda hypocrite : sinité, studios d'animation et discours orientaliste postmoderne}

\author{
PIERRE-MONG LIM
}

\section{Résumés}

Prenant pour objet deux dessins animés (Mulan et Kungfu Panda), la présente contribution tente de sonder la profondeur des différentes couches du message iconique proposé. Au sein de deux éléments récurrents, animaux anthropomorphisés et paysages, un même mécanisme de superposition s'exerce : celui du mythe (de la parole sur la « sinité ») et de sécurisation de l'identité du studio de production, exigence industrielle. De tels messages pointent, en vérité, plus loin que leur simple démontage sémiologique ; ils indiquent le lieu où continuent de se déposer les confettis du discours orientaliste, représentation immuable de l'autre et de l'ailleurs. Enfin, il nous faudra examiner en quoi ces médias de la culture populaire ne relaient pas seulement un discours traditionnel de l'orientalisme, mais soutiennent un phénomène qui voit « l'autre » dont il était question, se prendre pour tel, et tomber victime du prestige mythologique.

At stake in this paper, are the structures of two animation films (Mulan and Kungfu Panda) and the content of knowledge about China they unfold. I here intend to uncover the multiple layers of significations of the iconic message through two salient features: human-like animals and landscapes. A same mechanism is at work in both of them: on the one hand, a semiotic system of Chineseness, and on the other hand the will to answer commercial demand by securing the production studios' identity. Moreover, such an analysis of the system of significations is meant to display how orientalist discourse still finds a voice in those popular culture medias today. At last, it appears that 
the Other is now recapturing a discourse that once took him for signs, as a mean to speak of himself, hence blurring the habitual pattern of the myth.

\title{
Texte intégral
}

1 Tels cet acteur qui change de masques, tous différents sur un même visage, panda, tigres et dragons, peuple animal des films d'animation prenant pour cadre la Chine, endossent tour à tour les fonctions de signalement : des signes du mythe de la sinité à la « patte » du studio de production, tandis qu'en arrière d'eux se trame un même mouvement secret d'identification. ${ }^{1}$

La question nous guide ici de savoir comment, au travers de ces médias de la culture populaire, se poursuit, tout en se renouvelant, le discours orientaliste.

$3 \quad$ Y répond la parole de l'image animée, sous une forme apparemment légère et secondaire, les animaux ici mis en scène, et dans un décor signifiant. Ils se présentent à nous, porteurs d'une double charge symbolique qui sert en même temps que l'imaginaire sur la Chine, l'horizon d'attente de l'industrie de l'animation. A l'analyse de la mythologie d'une telle sinité animale vient se superposer celle de la rhétorique de l'icône aux fonctions fluctuantes, afin d'en dresser le diagnostic discursif, c'est-à-dire, la position de ces animations sur l'échiquier de la représentation de cet « autre nous oriental ».

\section{Sinité animale et paysagère}

Mushu et Po, voici nos deux interlocuteurs privilégiés au fil de ce texte. Le dragon malingre de Mulan et l'ursidé noir et blanc, un peu bêta, de Kungfu Panda : une telle présentation prête sans doute à sourire, ou à douter du bien fondé d'une analyse qui se veut tout à fait sérieuse. Mais nous entendons que c'est précisément dans ce type d'élément " inoffensif », secondaire ou apparemment évident, que les résidus tenaces du discours orientaliste sont venus se loger et continuent de séjourner. ${ }^{2}$ Pour cela, leur construction en signes répond à une structure qui mérite d'être analysée, en tant qu'elle révèle un ordre de parole très particulier, identifié comme mythe.

\section{Un masque mythico-sémiologique}

\begin{abstract}
La Chine est une chose, l'idée que pouvait s'en faire, il n'y a pas longtemps encore, un petit-bourgeois français en est une autre : pour ce mélange spécial de clochettes, de pousse-pousse et de fumeries d'opium, pas d'autre mot possible que celui de sinité. 3
\end{abstract}

C'est à la structure barthésienne du mythe, identifié comme sangsue du système linguistique de Saussure, que nous empruntons les termes théoriques du décorticage de la sinité animale. ${ }^{4}$

6 Rappelons très succinctement que « par-dessus » la triade linguistique signifiant-signifié-signe (mot), la parole mythologique se greffe, vide le dernier terme de la triade (le mot), le réduisant à l'état de signifiant du mythe, et y fait correspondre son concept. Comblée la profondeur de sens ; le signifié du mythe rend une surface plane et lisse, qu'il devient difficile de creuser. Le mythe joue de son ubiquité, de son constant alibi entre le sens et la forme, cette dernière 
servant de réceptacle au concept. Ainsi, la Chine, cinq lettres signifiantes " c-h-i-n-e » renvoyant à un univers en soi, se voit saisie par un concept (signifié) mythologique, qui l'aspire et finit par l'exhaler en sinité. ${ }^{5}$

7 Or donc, lorsque le neveu de Walt Disney, Roy E. Disney a, paraît-il, rejeté la première version de Mulan arguant que « Mulan est une belle histoire, mais où sont les grands dragons chinois et le folklore, les créatures mythiques? Quand on pense à la Chine une des premières idées qui vous vienne à l'esprit ce sont les montagnes brumeuses et des dragons de partout ", il exécutait devant nous le prestige mythologique : la Chine toute entière cachée dans un nuage. ${ }^{6} \mathrm{Du}$ chapeau tombe parfois un lapin -au gré des années seulement, mais plus souvent tigres et dragons, panda également.

De ces animaux que la répartition naturelle a dispersés au hasard des terres et des imaginaires, d'aucuns se sont vus ériger en figure solitaire, symbole d'un lieu, et, par métonymie, en sont venus à désigner l'autre oriental tout entier, vaincu par la multiplication des recoupements de réalités géographiques, littéraires, filmiques, qui l'ont réduit à tel signifiant mythologique animal. ${ }^{7} \mathrm{Car}$ nous le verrons, mythe et naturalisation sont d'un même tenant, et veulent faire oublier toute construction, toute action politique. ${ }^{8}$

Faute de place, nous ne pouvons dérouler tout le mécanisme à l'œuvre pour chaque " signifiant animal »; il y a certes l'évidence du dragon qui a accompagné presque tous les qualificatifs de " ce qui venait de là-bas » (pensons aux pays émergeants "les quatre dragons », les stars de cinéma comme « le petit dragon », les titres de films " la fureur du dragon », etc. la liste est longue). Une vieille et vague idée de l'Extrême-Orient, habité par des formes vidées, puis remplies flotte tout autour de lui.

Cependant, attardons nous plutôt un instant sur l'aubaine mythicosémiologique qu'est ce titre de Kungfu panda, concentré de "signifiants orientaux » auxquels s'ajoute une parole iconique double : un dessin (l'affiche du film) et un film (le dessin animé lui-même). Les signes du texte et ceux de l'image sont à mettre en rapport par un procédé que Barthes nomme la sémiologie iconique. ${ }^{9}$

11 Si nous soumettons l'image à une analyse du message qu'elle contient, nous verrons qu'elle fonctionne à la manière d'une publicité : il y a en effet un parallèle limpide entre l'affiche de cinéma (par laquelle on découvre pour la première fois le titre d'un film et son aspect visuel) et la réclame publicitaire. La publicité est un support qui facilité la tâche de l'analyse des signes, en ce qu'elle propose une image déjà pleine d'intentions, dont le signifié (le concept) doit être exprimé le plus clairement possible. Image et message (celui-ci sous-tend celle-là) entretiennent des rapports comparables à ceux du signifiant et du signifié. Barthes dégage de l'image trois messages, auxquels s'ajoute une dernière information (c'est-à-dire une indication quant au support : affiche, etc.). ${ }^{10}$

Le premier message est linguistique, il se trouve dans les signes écrits de la publicité (la légende, l'étiquette, le titre) : c'est un code qui utilise la langue. Dans certains cas le message peut exiger la connaissance de la langue ; pas dans le nôtre, ce qui met encore un peu plus l'accent sur le caractère préétabli de la parole et de la connaissance sur « la Chine », sinité. Car quoi de moins français que « Kungfu panda » $?^{11}$ En fait, ce qui ici paraît être une barrière de la langue n'est qu'un écran de fumée, car le lecteur (spectateur) « saisit » le titre sans réel besoin de compréhension textuelle. Ce qu'il ne connait peut-être pas, le 
kung-fu, est déjà une connaissance en soi : un mot étranger passé dans l'usage courant, qui désigne une pratique orientale. En ce sens, le message de l'affiche, par l'exotisme du texte, annonce son intention de dépaysement, sa promesse d'Orient. Il est vrai que pour un découpage analytique plus rigoureux, on devrait supposer une distinction dans l'ordre des messages (ce qui tient du texte pur et ce qui tient de l'intellection), mais faute d'espace, nous tablons sur le fait que le lecteur de l'affiche perçoit simultanément les signes. On le peut, car on va le voir, un tel message linguistique est soutenu par une image et un discours d'une puissance uniformisante dont l'efficacité est redoutable.

A ce premier message linguistique se surajoute un autre signe, qui contient en lui l'idée prête à être véhiculée par les consonances et l'imaginaire qui gravitent autour du nom : c'est le concept qui sous-tend tout le reste du message (là l'italianité, ici la sinité, etc.). « Kung-fu panda », le titre signale une intention "grosse comme une maison », une intention de sinité : le balisage de la lecture de l'affiche en est presque redondant, à la limite parodique. Le titre se déchiffrerait de la manière suivante $:$ Kungfu $=$ Chine $;$ Panda $=$ Chine, un titre qui, dans l'imaginaire, redouble sa parole : Kungfu Panda dit « Chine Chine » ou mieux, « sinité-sinité ».

En outre, cette lecture s'accompagne d'une image. Les deux autres messages sont d'ordre purement iconique : il serait en principe difficilement envisageable de distinguer les deux aspects suivants, étant donné que le spectateur les reçoit en même temps. Mais Barthes pour les besoins de la clarté de l'analyse sémiologique de la structure de l'image (comme en linguistique le signifiant n'est en pratique jamais séparé du signifié) justifie cette séparation, de plus, elle prépare l'explication du rôle social de l'image.

Tout d'abord, on sait qu'à un niveau purement conceptuel, il existe une image toute pure, où figurent les objets « en eux-mêmes » qui peuplent la scène (sur l'image à laquelle nous nous intéressons, ce serait l'animal en lui-même, pris dans une sorte d'innocence zoologique). Ils ne répondent à aucun système de signes puisqu'ils se signifient par eux-mêmes. Ce message n'est donc pas codé, il est un message brut. Celui qui reçoit ce dernier n'utilise en fait aucun « savoir », mais une faculté de perception de l'objet : il le voit et l'identifie. Barthes qualifie ce savoir "d'anthropologique », car contrairement à la parole iconique connotée, celle-ci est littérale et non symbolique : c'est l'image dénotée, le signifiant iconique.

16 Le second niveau de réception répond à un système de signes plus élaboré. La scène représentée envoie des signaux que le spectateur capte en fonction de ses propres connaissances, de son savoir : en cela, les domaines auxquels sont rattachés les objets peuvent être variés (culinaire, cinématographique, etc.) ; d'autre part un savoir d'ordre " culturel », qui s'accorde au premier signe linguistique : c'est la conscience plus « localisée » dans laquelle les signifiants sont ancrés (cependant, une limite mérite d'être introduite ici, puisqu'un tel savoir localisé, qui ne parlerait pas de la même manière au spectateur français, américain ou chinois, est de moins en moins vrai, à cause justement d'une propagation de supports identiques de l'imaginaire). En suivant donc le sillon tracé par l'analyse mythologique, nous pouvons reprendre à notre compte ce système de signification afin d'expliquer l'affiche de Kungfu Panda.

On le voit, il y a dans celle-ci une certaine densité de l'image, qui se signale sur plusieurs niveaux : le support ludique animé, l'univers pelucheux de l'enfance, l'art martial et les icônes cinématographiques " asiatiques ». 
Distinguons une multitude de couches, que le regard du spectateur épluche au gré des références qu'il y associe.

A un degré tout à fait basique, le style graphique de l'affiche (et qui dit style, dit connotation) se place d'emblée dans le monde du dessin animé. Une première couche, peu intéressante dans notre cas, recouvre ainsi les personnages présents sur l'image : ce sont des héros de dessin animé. C'est peut-être celle que va saisir un parent en quête de divertissement pour son enfant (ce dernier est lui aussi susceptible de capter une image, celle du gentil nounours en peluche par exemple) : au milieu d'autres affiches publicitaires, celle-ci envoie les signes d'un support, le film pour enfant.

Cependant, le dessin prive définitivement l'image de son sens dénoté : clairement, de par son travail, son apprentissage, son parti pris même, le dessin comme le dessin animé renoncent d'emblée à la possibilité d'une lecture pure, plagions Barthes, d'« une lecture au degré zéro » de l'image. Ainsi, le panda « anthropomorphisé » de l'affiche ne ferait-il que lever la jambe ? L'image dénotée voudrait que oui : il effectuerait un simple mouvement de contorsion animale, ou peut-être même un trouble physique du comportement du panda, ou encore un coup de pied, etc. tout reste donc possible dans l'interprétation du geste. Mais le panda qui projette vigoureusement sa patte, les bras dans une position peu commune, sous le titre « Kungfu panda », fait bien plus que tout cela : il signifie. Il signifie la "martialité ». Signe entouré par d'autres, qui viennent en renfort (ou à la rigueur, le laisser tel quel, selon le regard du lecteur-spectateur) ; l'imaginaire extrême-orientaliste déballé dans ces signes : les baguettes, le « dimsum », le petit panda habillé de la longue tunique du sage chinois, les mains rentrées dans les manches; et le fond de l'affiche, gaffe orientaliste, irradié des rayons d'un soleil nippon, etc. Tout ce savoir culturel farci dans ces messages iconiques, porte en lui une promesse divertissante de l'Orient.

20 De ce fait, le lien causal s'inverse, et donne la relation suivante : on se trouve en Chine parce qu'il y a le panda ; ce sont des arts martiaux chinois parce qu'il y a un tigre (et non, il y a un panda parce qu'on se trouve dans une certaine partie du monde...) à partir du moment où notre savoir ne s'appuie plus sur la réalité, le contexte géographique ou historique pour expliquer les phénomènes, on peut dire que le mythe opère pleinement, la combinaison de sa forme et de son concept sont tout à fait superposés et invisibles. Le rapport à la nature se trouve renversé, bouleversé. ${ }^{12}$ Au niveau idéologique, la communication de masse repose en grande partie sur ces jeux de substituabilité de l'image (dénotée, naturelle, essentielle) à l'idée construite, l'histoire, ou le récit.

Ces quatre signes forment donc l'ensemble de ce que Barthes nomme le message iconique : " ils forment un ensemble cohérent, car ils sont tous discontinus, obligent à un savoir généralement culturel et renvoient à des signifiés dont chacun est global $» .^{13}$ Le point nodal de lecture se trouve dans l'intrication étroite entre les trois types de messages.

22 Et la présente rhétorique de l'icône pourrait se décliner, à quelques nuances près à tous les animaux/personnages présentés par ces animations, qui se nourrissent et nourrissent en retour, cette sinité animale. Nos protagonistes, créatures de ce mécanisme parasite, sont plongés dans l'élément mythologique ; ils n'en ressortiront que pour une nouvelle fonction de signalement. 


\section{Dans le décor}

Nous nous sommes arrêtés sur la caractéristique animale de ces animations, mais il nous faut signaler un autre composant, le paysage, qui répond en écho à la naturalisation de la sinité. Le " décor ", essentiel à l'économie du dessin animé, puise pareillement dans un imaginaire de signes et de mythes, notamment dans la peinture de paysage «traditionnelle ».

24 Notons d'emblée le poncif visuel par lequel s'ouvre par exemple Mulan. Le générique débute par les touches d'un pinceau imaginaire formant çà et là quelques pins à flanc de montagnes, puis une longue construction, la Grande Muraille. Cette présentation signale deux choses : une géographie (la Grande Muraille ne peut être ailleurs qu'en Chine) mais aussi un temps, car la calligraphie et la peinture forment un couple de références à ce qu'on imagine être la Chine traditionnelle, un passé présent.

25 Le début du récit pose le pied sur la Grande Muraille même, où les troupes sont attaquées par le Shan Yu et sa « horde sauvage ». ${ }^{14}$ Mais cette utilisation du symbole architectural, plutôt fine, sert à autre chose qu'établir une réalité historique : là encore, elle pose essentiellement une balise pour guider l'imaginaire. Si l'on fait rebondir cette rhétorique de l'image sur un film intitulé Mulan, constellé de gouttes d'encre de Chine, et qui s'ouvre sur la Grande Muraille, on comprend que, dès le début, l'imaginaire se trouve bien calé au fond de son fauteuil de stéréotypes.

26 Si la Grande Muraille n'est pas à proprement parler un paysage de monts et de fleuves, on ne risquait rien en pariant que ces éléments viendraient ponctuer l'action du film : ainsi comme le voulait Disney neveu, les brumes vaporeuses étendent leurs bras autour des montagnes ; tel un des ces rouleaux d'un quelconque «peintre chinois » dont les paysages sont la réminiscence. Tout doit être signe : la floraison des fleurs de prunus, les montagnes que couronnent les nues, le fleuve qui serpente dans la vallée, ces éléments se doivent de signifier la Chine, ou mieux, ils expriment l'idée d'une certaine Chine, la sinité, celle de « Mulan ».

27 Les décors de Kung-fu Panda sont eux aussi saturés de ces paysages brumeux d'où jaillissent les têtes nues des montagnes ; çà et là, des arbres aux branches chargées de fleurs. Certaines scènes semblent n'avoir aucune incidence sur le déroulement de l'intrigue, pourtant elles ont une importance toute particulière dans leur fonction de « localisation » : ce sont des paysages isolés, intercalés entre deux scènes d'action (par exemple, après que Po a été violemment corrigé par le maître ou ses condisciples) : à ce moment précis, le paysage n'est plus un arrière plan, mais, il est pris pour lui même ; serait-ce alors un tableau de "shanshui » en images numériques ? $^{15}$ Tout y est, un peu trop même : les montagnes dans la brume, le fleuve dans le creux de la vallée, puis le soleil qui se couche à l'horizon... les animateurs vont jusqu'au bout du fantasme orientaliste, mettent bout à bout : la pagode à flanc de montagne, signe architectural de la sinité par excellence, le temple du kungfu qui se devine entre un nuage qui s'effile, les mille marches qui mènent au temple (on remarque que les chiffres qui quantifient « l'Orient » sont la plupart du temps astronomiques : mille marches, des milliers d'années, l'armée des dix mille guerriers, on peut diagnostiquer une mise à distance exotique, tant géographique que temporelle) ; cette redondance de " connotateurs iconiques »ne laisse aucune chance à une éventuelle lecture alternative du 
paysage. ${ }^{16} \mathrm{Si}$ bien qu'on penserait presque à une parodie, la vision caricaturée de la Chine au travers du « film américain ». ${ }^{17}$

Néanmoins, tout autre nous semble être la fonction de ce paysage : une fois réinvesti le contexte du dessin animé en lui, on comprend qu'il sert essentiellement de frontière à la perception ; c'est une sorte d'image de guide touristique qui revient comme un leitmotiv au cours du film. Le spectateur ne doit pas oublier le lieu de l'action ; dans l'ambiance d'une Chine traditionnelle et vaporeuse. Les animateurs, le scénario, s'évertuent à garder vivante l'illusion, car l'ambiguïté du rôle des animaux tout comme le pseudo kung-fu, tout comme la pseudo sagesse orientale ont le tort de jouer sur les deux tableaux (vision occidentale-sinité). Les monts et les fleuves qui s'interposent témoignent du besoin de ce moment d'arrêt et de remise en condition, car absolument aucun autre élément du film ne peut continuer à faire se sentir le spectateur en Chine : le panda est finalement plus Coca que thé vert, et les nouilles parfum « Mac Do »; non décidément, le kung-fu est devenu trop international, inauthentique. ${ }^{18}$ De là comment réaffirmer une sinité ? Par le paysage et sa référence à une tradition picturale, qui plus que jamais dans ce cas est mythologique.

Ces interludes paysagers ne sont donc pas un hommage ou une tentative d'hybridité avec la peinture chinoise, ils fonctionnent comme des rappels géographiques : ils ne cadrent plus l'action ; isolés, ils la recadrent.

Pour résumer, au niveau d'une sémiologie de l'image, montagne et eau sont positionnées en tant que signifiants, ils signalent " physiquement » ces éléments. De la même manière que dans la partie précédente, ces signes se confondent avec une idée du paysage chinois, notamment la peinture de paysage. Dans ce mouvement, le paysage et la peinture ne sont plus envisagés séparément : ils n'existent plus pour eux mêmes, mais pour l'ambiance qui se crée autour d'eux. L'utilisation s'apparente au mécanisme du mythe qui déforme tout à fait les significations possibles, du paysage et de la peinture, et n'en donne qu'une signification décorative. En ce sens on peut dire qu'il agit telle une naturalisation de ce paysage en peinture, car à aucun moment, le spectateur n'est autorisé à penser qu'un paysage pourrait ressembler à autre chose que cela. En superposant parfaitement la peinture à la réalité du décor, le sens s'en trouve totalement dévié : la Chine est ainsi un tableau par essence, la peinture ne se reconnaît plus comme la projection extérieure d'une vision personnelle, mais comme la seule vérité du paysage chinois. Ce qui aboutit d'une part à figer la Chine dans ces traits d'encre, et d'autre part à la présenter comme un spectacle. La dépossession est ici double : la peinture est complètement vidée de son sens et, de par ce vide, cette déviation du sens, on tend à faire apparaître ce qui ne devrait être qu'une peinture comme la réalité. Le paysage chinois, encore une fois, apparaît indiscutable et imperfectible, «tel qu'il est ", une Nature : il faut donc plutôt parler de " ressemblance » à ce paysage essentialisé, plutôt que de "référence » à une peinture passée dans le domaine de la Nature.

31 L'" espace du rêve » dont il est parfois question, n'est censé désigner que la peinture et les rapports que l'homme entretient avec elle. ${ }^{19}$ Alors que dans le cas présent, le rêve est transmué en réalité : mais c'est là le spectacle de la réalité, un tableau reconstitué par le fantasme de l'ailleurs. ${ }^{20}$

Cette image d'Epinal chinoise, pagodes, montagnes et eaux, prunus en fleur, finalement, nous renseigne sur un temps autant que sur un espace : au delà du 
simple lieu, il faut voir dans ces appels au «shanshui », un « discours fleuve », dont le cours temporel s'est suspendu.

\section{Double dragons}

Les artifices du mythe illuminent un temps le ciel de la sinité ; retombés dans le contexte de l'industrie de l'animation, ils se font signes à nouveau, pour une cause différente : l'identité du studio.

\section{Disney : les compagnons anthropomorphes}

Mushu le dragon, gardien déchu de la famille de Mulan, fonctionne sur ce même mode sémiotico-mythologique, et signale donc la Chine.

Nous avons pourtant laissé entendre que ces animaux portent des masques : sous celui de la sinité, Mushu en dissimule un autre ; bientôt, il change de visage et endosse une nouvelle fonction, celle du personnage-signe de Disney.

Car le rôle de " compagnon-bouffon " renvoie à toute une tradition des dessins animés du studio, qui tient à conserver une sorte d'identité, marque de fabrique qui estampille chacun de ses longs métrages évènements. En fait, l'utilisation de ces animaux « anthropomorphisés » remonte à près d'un siècle, aux débuts même de Walt Disney dans l'animation. ${ }^{21}$

Dans le projet original d'ailleurs, Mushu devait s'annoncer par une chanson typiquement américaine, la formule Disney du cocktail de formes et de concepts : « le bien contre le mal, de l'émotion, des chansons accrocheuses, des animaux drôles et mignons qui accompagnent le personnage principal, le doublage des voix par des acteurs, etc. ${ }^{22}$ L'introduction des compagnons, Mushu en tête (et le Criquet), se fait ainsi par la voie disneyenne ; sans oublier que l'histoire de Mulan (à l'origine, une ballade que l'on fait remonter environ au $3^{\text {ème-4}} 4^{\text {ème }}$ siècle) connait certes quelques variantes, mais il n'y est jamais fait mention d'un dragon et d'un criquet qui accompagnèrent la courageuse jeune fille, ou de quelconques gardiens de la famille. ${ }^{23}$ Les réalisateurs peuvent s'emparer de la trame du récit poétique, sans craindre pour autant la rigueur historique et remodeler sur les imaginaires de la sinité et du studio : en plus de signes orientaux qui parlent à cet imaginaire, " un criquet et un dragon rendaient l'histoire plus intéressante ». ${ }^{24}$

Cependant, encore une fois, on le voit, ce n'est que la forme qui est présentée. Or une telle forme, on le sait de par le système mythologique barthésien (qui désigne par ce terme le premier moment du schéma mythologique), ne sert que le concept et la signification. ${ }^{25}$ Derrière elle se tient le mythe, à couvert. Ainsi Mushu, en tant qu'il fonctionne comme signifiant de ce mythe, se trouve tout rempli de sinité, d'imaginaire sur le dragon chinois, mais simultanément, il complexifie ce système puisqu'il porte lui-même un masque : idée de sinité déguisée, aux ordres du propos proprement disneyen du film. Le coréalisateur déclare également à ce propos : «(...) nous n'allions pas faire un dessin animé chinois. Nous ne le pouvions pas. Nous ne sommes pas chinois. ${ }^{26}$ En se libérant de la gangue de "l'authenticité », il devient tout à fait acceptable pour les réalisateurs d'appliquer la formule floridienne des " animals sidekicks », livrant ainsi un produit purement hollywoodien ; et voilà pourquoi de dragon 
chinois fort respectable, Mushu tchatche désormais en argot afro-américain, doublé par l'acteur Eddie Murphy. ${ }^{27}$ Oublié le puissant animal mythologique, symbole de force et de prospérité, place au dragon " has been ", qui pour combler sa petite taille et son souffle de feu limité, s'emploie à quelques artifices pyrotechniques.

Pour l'équipe de production comme pour le public, il est crucial d'identifier les fonctions de ce personnage, son articulation dans l'interaction avec l'histoire et les autres protagonistes : le rôle de Mushu est avant tout clownesque, il est le compagnon qui provoque le rire, un gaffeur au grand cœur (souvenons nous que c'est lui qui brise le " grand dragon de pierre » supposé accompagner Mulan dans sa tâche ; plus loin, incapable de contrôler sa flamme -embêtant pour un dragon- il détruit la réserve de munitions de la garnison, etc.). Personnage-type (et typiquement) des studios, dont les défauts sont la plus grande richesse, Mushu comble l'horizon d'attente du public disneyen en tant qu'il est placé pour générer et attirer la sympathie.

Bref, le dragon demi-portion a décidément fort à faire : gardien à temps plein des « ancêtres chinois » (et par là, de la sinité) puis de la tradition du studio floridien, son masque comme sa casquette, sont doubles. ${ }^{28}$

\section{Le surpoids de l'anti-héros}

Les studios Dreamworks n'ont pas encore acquis la notoriété du « grand frère » (ennemi) Disney. Il est sans doute un peu tôt pour parler d'une « formule Dreamworks » comme on le fait pour Disney. ${ }^{29}$ Il n'en reste pas moins que dans les films d'animation à succès de Dreamworks, certains traits apparaissent et réapparaissent, de façon récurrente. A bien y regarder, deux aspects se répondent en écho : l'irrévérence et la figure de l'anti-héros.

Après Shrek, comme après Bettelheim, on ne verra plus les contes de fées comme avant. Cet anti-héros d'ogre, cynique, asocial et peu hygiénique, campe en quelque sorte l'identité fondatrice de ces studios d'animation; le " héros malgré lui » entraîne à sa suite une petite foule d'épigones animés. ${ }^{30}$

Dans Kungfu panda, Po répond en tout point au besoin d'un tel personnage : il se trouve affublé de bien des tares par rapport au héros chevaleresque classique ; non content d'être légèrement obèse, couard et disons-le, pas très futé, notre panda est la risée de ses compagnons, le souffre douleur du maître aigri et l'objet du choix douteux d'une vieille tortue taoïste atteinte de gâtisme.

Cette fois-ci certes, rien de très neuf dans l'iconoclastie, car la " comédie kung-fu » est déjà passée par là (le maître loufoque et excentrique -en la personne de Wu Gui, le maître tortue taoïste- de même que la petite taille et l'aspect chétif du maitre de Po, sont des mécanismes plutôt classiques ; de même que Po, figure du disciple indiscipliné, empêcheur de tourner en rond, finalement, ne remet rien en question, si ce n'est l'a priori sur les " gros », démenti plus tôt, d'ailleurs, par l'acteur hongkongais Samo Hung). Même si les coups de griffes du panda écorchent moins le mythe enfantin que les blagues à double sens de l'ogre vert, cela ne retranche rien au mécanisme du masque que nous évoquions à l'instant ; la ligne directrice comique du studio se poursuit dans cette animation.

45 A nouveau, il semble qu'il faille y voir un système de signes « doublé », où les références à un imaginaire de sinité (comme on l'a vu plus haut avec cette parole iconique) servent, ailleurs et en même temps, les visées propres à la 
pérennité de la marque Dreamworks.

\section{Prolongements orientalistes}

Le visage du discours orientaliste transpire sous les deux faces explicitées des personnages animaux animés : en une constante (re)création de $l^{\prime}$ (extrême)Orient, au travers de signes transformés en essence, la "nature orientale » perdure dans le miroir occidental et ouvre un nouveau passage dans l’imaginaire culturel populaire local désormais globalisé.

\section{Cinquante ans, une éternité}

47 Formulé dans les propos de Roy Disney, « mot d'esprit » révélateur de l'inconscient orientaliste, l'imaginaire occidental du divertissement ne conçoit pas une Chine sans ces animaux, cette ambiance empreinte de mysticisme : pas de représentation en dehors de tels éléments, que le mythe a en quelque sorte " naturalisé » chinois. Les types orientaux définis une fois pour toutes, et en cela imperfectibles et indiscutables (on l'a vu le rapport causal est inversé) ont investi la sphère de l'essence : c'est ainsi que de 1958 à 2008, aucun choix divergent n'a pu être envisagé.

48 Quarante ans avant Mulan, la Toei (un studio d'animation japonais) réalisait Le serpent blanc : il est tout à fait frappant d'y trouver, à très peu de chose près, les mêmes ressorts de signes. Avaient-ils réellement quarante ans d'avance ou alors, est-ce Disney qui est en retard d'autant, au moment de Mulan?

49 Comme le note Gregory Lee pour Mulan, le film réinscrit la Chine dans un discours d'immobilité pré-moderne, geste réédité par toutes les autres animations qui prennent pour cadre la Chine. ${ }^{31}$ Que la légèreté du prisme « animal animé » ne nous trompe pas, nous sommes en présence d'un très typique "discours orientaliste », qui opère ici sur la Chine, de par l'idée de sinité. $^{32} \mathrm{Si}$ par exemple le dragon métonymise constamment la Chine, nul hasard à cela, car l'idée qui l'accompagne va de pair avec une représentation (a)historique (et on verra dans un instant que le discours auto-orientaliste s'en nourrit tout autant).

Et si l'on peut sans doute nuancer la véhémence de la critique que Ziauddin Sardar fait de Disney, il nous faut reconnaître avec lui la présence et la diffusion de la parole orientaliste par le biais des mass médias et de la culture populaire, porteuse de ces stéréotypes immobilisant l'autre. ${ }^{33}$

51 Le couple, exotisme spatial et temporel, va main dans la main : l'Orient des représentations promet d'être un endroit merveilleux et sensuel, pour " une escapade exotique ». ${ }^{34}$ La lointaine Chine traversée de ces « hazy mountains », peuplées d'étranges animaux renvoie à une Chine plus rêvée que réelle, et qui pourtant paraît plus authentique. L'idée était évoquée plus haut : les paysages trahissent la fixité historique dont ils sont les témoins muets ; la grandeur passée, l'un des thèmes les plus retors de l'orientalisme tel qu'il est envisagé par Said, saisit un Orient immobilisé, qui n'existe au présent que dans sa forme passée, ses montagnes sont sa prison temporelle.

52 Se défaire de telles représentations de paysages exigerait un arrachement du cercle d'auto validation de ces références paysagères qui, d'un dessin animé à 
l'autre, s'étirent en filigrane. Depuis Le serpent blanc, et plus encore depuis Mulan, jusqu'à Kungfu Panda, le spectateur attend la Chine et ses montagnes enchaînées par les nuages : ressemblances aux peintures, puis ressemblances entre animations, et ressemblances de ressemblances, le cercle est bien clos sur lui-même. L'espace se trouve effectivement changé en temps, il le fige dans les " temps immémoriaux » au moyen du mythe des montagnes et des eaux, configuration physique de l'immobilisme. L'idée d'un tel passé est retenue captive dans ces formes et se trouve constamment actualisée : « immémorial » donc, ce lieu dont la mémoire immédiate est sans cesse effacée, substituée à un souvenir imaginaire.

L'immobilité, sœur de l'aphasie, scelle l'image de l'autre et de son ailleurs, et les tient silencieux en s'exprimant pour eux : au cours de ce processus le dessin animé, identifie les éléments vitaux, les éléments types de la culture de l'Autre, puis les décontextualise, les vide de leur histoire afin de mieux les reconfigurer selon sa compréhension propre. La mise en contexte paysagère retient un " parfum chinois », et situe son histoire uniquement autour de symboles (Grande Muraille, pics, grottes de Bouddha, etc.). L'espace « chinois » est vide de sens, incapable de communiquer, il ne semble pouvoir s'exprimer que par procuration, via une cette fenêtre temporelle (ahistorique) et spatiale. ${ }^{35}$

\section{Authenticité et spectacle}

Au service du désir occidental, l'(extrême) orient miroite sur le grand écran, en un reflet sans défaut. Et de loin, l'Occident préfère sa créature orientale à une réalité différente de ce que pourrait être cet ailleurs : son aveu d'amour propre s'exacerbe au moment même où le simulacre de la réalité se fait toujours plus intense.

Dans la lignée orientaliste, le regard de l'animation refuse de s'accommoder sur un autre plan que le sien. Dans cette optique, il est d'ailleurs intéressant de noter que Mulan est réalisé dans le contexte historique américain d'une tradition de récupération et de réinvention de la sinité. ${ }^{36}$ Le film lorgnait certes du côté du marché chinois, mais fût néanmoins récupéré par la culture populaire orientaliste américaine. Cela s'explique par le fait que les produits culturels « locaux » sont plus compétitifs pour des raisons de proximité et de familiarité du public, notamment avec la langue et le contexte culturel dans lequel il s'inscrit. ${ }^{37}$ Dès lors, on comprend que le système de représentations répond également à des exigences que l'on pourrait qualifier d'industrielles. ${ }^{38}$

La marchandisation de la relation à l'ailleurs entache la réalisation d'un procédé pseudo-hybride. Elle suit un dosage uniforme : une part de spectacle affiché (reconnu, c'est la « patte » du studio) et une part d'authenticité (comprenons : au spectacle de « l'authentique »). On sait que des équipes de réalisation furent déployées en Chine dans cette quête d'authenticité. Ce trait orientaliste bien connu va faire correspondre sa parole à l'objet oriental et confirmer l'idée d'une Chine ancestrale ; le besoin d'authenticité cherche désespérément la légitimité, une identité, une essence, une pratique historique ; mais où trouver tout cela ? Dans l'ancienneté (toujours donc, la convention de ce qui est ancien), dans les pratiques passées ; et de remonter alors le long des «traditions culturelles » (dont la peinture de paysage est un exemple). Mais si dans les dessins animés, la peinture évoque la « différence » chinoise (" mon autre exotique »), elle émane cependant d'un média 
occidental : en ce sens, la sinité se fait présente par une sorte d'absence chinoise, représentée ailleurs, elle n'en retrace pas moins les signes de la sinité, une sinité d'apparence, de spectacle. ${ }^{39}$

Cette démarche répond au double mouvement décrit plus haut : de parler pour l'Orient (mutisme), et à l'intérieur de cette parole, d'y faire correspondre un espace et un temps passé rêvé (immobilisme). Ainsi, l'œil comme l'imaginaire du spectateur, éduqués pour superposer cette image pour ce sens, valident encore un peu plus le discours sur l'Orient.

Depuis peu, on a assisté, comme par étapes, à une universalisation de ces significations, ou plutôt, à une globalisation (« hollywoodisation »). Tout d'abord par un moyen qui peut paraître paradoxal, puisqu'on a parlé de « localiser » le produit, le rendre accessible aux systèmes de référence dans les autres régions où le produit s'exporte : ainsi, Disney et Dreamworks « localisent » leurs stratégies marketing. Ces stratégies consistent par exemple à utiliser des stars et acteurs locaux pour le doublage de voix des personnages : c'est ainsi que dans le Mulan version chinoise, Jackie Chan a été engagé pour doubler le général Chang et pour interpréter la chanson du héros traditionnellement présente chez Disney. Mais il semble qu'aujourd'hui cette technique de " glocalisation » soit engloutie par une homogénéisation toujours plus radicale : fait significatif, dans Kungfu Panda, Jackie Chan n'est plus engagé pour doubler la voix en chinois mais tout de suite en version originale anglaise. ${ }^{40}$ Faire la promotion du film avec Jackie Chan c'est assurer un semblant d'authenticité, ainsi chargé de signifier la Chine, et pas n'importe laquelle, le royaume ancestral du kungfu (avec ses techniques basées sur des mouvements d'animaux, etc.). Il nous revient ainsi en mémoire la première mondiale de Kungfu panda au Festival de Cannes de l'année 2008 : durant quelques jours, la ferveur autour de ce qui « faisait chinois » (toits de pagodes en carton pâte, singeries kung-fu, nems et compagnie) prenait un tour burlesque. Les ambassadeurs de cette Chine ? Des acteurs hollywoodiens dont il n'est pas utile de citer ici les noms, et s'il faut parler de « MacDonaldisation » du cinéma, c'est assurément autour de ce type d'événement. La vision orientaliste spectaculaire tourne à plein régime dans l'industrie du divertissement, où plus que jamais l'Orient est un lieu de pur plaisir, consommé à la satisfaction..$^{41}$ La manœuvre promotionnelle à l'œuvre, archétypale d'un monde reposant sur les fondements du spectacle, répond point par point au qualificatif «fallacieux $» .{ }^{42}$ Non sans ironie, Gregory Lee faisait remarquer que Mac Donald n'a pas eu à créer son "MacPekingduckburger » pour pénétrer le marché chinois. ${ }^{43}$ De local, les studios Dreamworks n'auront eu, quant à eux, besoin que de l'acteur Jackie Chan.

Difficile pourtant de croire une minute à la supercherie : l'acteur hongkongais, qui double un personnage presque muet (cherchez l'erreur), là encore, fonctionne comme un signe ; mais un signe du spectacle. Guy Debord avait annoncé une division des tâches de moins en moins nette : «c'est dans de telles conditions que l'on peut voir se déchaîner soudainement, avec une allégresse carnavalesque, une fin parodique de la division du travail $»{ }^{44} \mathrm{La}$ parodie est ici tout à fait exemplaire. Outre la -trop- grosse ficelle marketing du doublage des voix, qu'en est-il du rôle cinématographique de l'acteur, dont les répliques se comptent sur les doigts d'une main (et moins encore, c'est dire) ? Le spectacle ne demande en rien à Jackie Chan une telle performance, c'est un 
tout autre type de représentation qu'on attend de lui : « son statut médiatique » et sa charge d'authenticité chinoise. ${ }^{45}$

La persistance du discours orientaliste populaire s'étoffe donc par divers mécanismes, tant dans les médias occidentaux, que par un phénomène d'intériorisation du discours par la Chine elle même (d'ailleurs le film Kung-fu Panda cette fois a été un franc succès dans les salles de cinéma chinoises, battant le record des recettes d'entrées pour un film d'animation, et plutôt bien accueilli par la critique) : l'assimilation à cette globalisation, pose la question de certaines problématiques autour du « postmoderne et de l'Autre ». ${ }^{46}$

\section{Stade du papier glacé : l'autre, mon hybridité}

61 Lorsque Po reçoit « le rouleau du guerrier dragon » censé lui conférer le savoir ultime sur les arts martiaux, quelle stupeur ! Une fois déplié, pas un mot d'inscrit sur le rouleau. On s'interroge, on s'agace, on accuse feu la tortue gâteuse. Que doit-on comprendre là ? Une réponse zen à la soif démesurée de connaissance, à la vaine ambition du panda ? Le propos voudrait nous fait croire à la page blanche, à une étendue des possibles. Mais le rouleau se révèle n'être qu'un miroir, qui nous reflète un " tel quel ». La tautologie du panda, " toi c'est toi ", " moi c'est moi ", est le fondement même du mythe et de l'univers orientaliste qu'on vient de décriren: « la tautologie fonde un monde mort, immobile ».47 L'image coupe court à toute explication, toute correspondance se doit de tomber sous l'évidence.

$\mathrm{Si}$ l'on en croit Ziauddin Sardar, c'est également le propre du postmodernisme phénomène continu de la domination occidentale sur son double oriental. ${ }^{48}$ Car c'est bien de cet « autre moi -occidental- » dont il s'agit. Said, qui dans ses premiers écrits applique l'équation du savoir au pouvoir à l'exercice de la représentation de l'Autre, évoque l'absence, l'aphasie, l'inexistence de l'oriental.

Dessin animé oblige, la formule magique existe bel et bien, elle reprend même les paroles de la méchante reine : « miroir, ô miroir, dis moi... », Po ne posera pas la suite de la question, mais simplement « qui suis-je ?», et si l'on se permet de pousser un peu plus loin la glose sur le reflet, on entendra la fin de la réponse : sois toi même, un panda chinois cool, qui aime le coca et les nouilles ; tu ne bouges pas depuis plus d'un demi-siècle, mais c'est ton sort, " tu es toi $\gg .49$

64 Le monde compris sur le mode réflexif construit un autre moi ; l'image renvoyée c'est l'Occident qui se perd, se cherche, cherche des réponses ailleurs, chez l'Autre, puis lui fait correspondre ses attentes, et ne propose finalement qu'une énième réflexion centrée sur l'Occident (dans le cas présent, l'industrie culturelle hollywoodienne). Le monde est peut être bien devenu un relatif absolu dans lequel ne subsiste plus de vérité, où tout est égal ; sauf que nous posons toujours ce qui a un sens et ce qui n'en a pas.

Ainsi, alors que Joseph Chan parlant de Mulan, évoque les aspects d'hybridité (métissage) entre ancien et nouveau, traditionnel et moderne, Orient et Occident, etc., nous émettons pour notre part de fortes réserves. ${ }^{50}$ Outre une problématique de fond qui touche au débat sur le concept même d'hybridité, dans les antres du spectacle on se targue bien vite de ce multiculturalisme ou multi-ethnicisme culturel, tandis qu'on se contente d'en envoyer les signes. La duplicité du masque telle qu'on l'a décrite plus haut, 
trahit (à peine) et révèle l'intention véritable : en envoyant les signes de métissage, les studios passent à leur propre aveu d'incapacité à se fondre avec l'ailleurs qu'ils ont pris comme objet, et se trouvent forcés de placer des signes visibles. On voit revenir l'opération du mythe. Prenons pour exemple la référence aux peintures de paysage : fadeur, suggestion, compris comme la quintessence des « codes chinois » picturaux, ne sont pas réincorporés, mais montrés ; à trop vouloir être suggestif, l'intention finalement s'explicite : le dessin animé cherche à nous montrer qu'il utilise ces codes chinois. Encore une fois, comme le veut le mythe, on peut être trompé par le niveau de lecture : le paysage suggestif ne l'est qu'en apparence, il envoie les signes de la " suggestivité ». Ainsi, Mulan n'est pas un dessin animé américain mêlant dans ses aquarelles le noir de fumée, il est une compréhension, et la manifestation de cette compréhension, de ce qu'il entend être « l'art chinois » au service de l'authenticité.

En sorte que, cette «politique » des studios que l'on pourrait presque qualifier de postmoderne dans son désir de pluralisme, ne fait jamais qu'envoyer les signes de l'ailleurs, sans jamais pouvoir vraiment se dépasser : « tout objet non-occidental apparaissant en Occident, est strictement désigné à servir une mode ethnique ou fait office de symbole vide $" .{ }^{51}$ A supposer donc que l'hybridité soit une réalité, dans de tels films elle s'arrête sur les bords du stéréotype, et ne franchit pas le pas vers une conscience de l'existence de l'Autre, de sa différence : une telle reconnaissance ou l'espoir de diversité culturelle passent éternellement au tamis du filtre des signes et du mythe. ${ }^{52}$

Et l'application d'un simple principe de production-reproduction, là encore, veut faire croire à une nouveauté, à l'ouverture d'un océan de possibilités ; mais on le voit, le renouvellement est tout au plus technique (le passage au dessin d'animation numérique), et rien de plus.

Ce processus d'uniformisation plus que de "transculturation ", " une forme d'emprunt culturel, par lequel une culture en reconfigure une autre, pour ses propres besoins ", aboutit à la reconstruction du discours colonial, remarquable dans sa capacité à s'approprier les êtres et les imaginaires. ${ }^{53} \mathrm{Si}$ la colonisation passe désormais par d'autres biais que l'occupation manu militari du territoire, aujourd'hui, un sens de communauté collective imaginaire réunit les populations qui sont «toutes représentées » : le pluralisme postmoderne annoncé n'en reste pas moins contrôlé par quelques organismes, qui seuls décident de ce qu'est la pluralité et de comment elle se représente. Le mouvement est d'ailleurs plein de malice : une fois assimilés et consumés par l'Occident, les produits culturels «pluralistes » sont ensuite réexportés vers d'autres pays (ce que, par commodité, on peut nommer «le monde non occidental ", terme employé par Sardar, mais du reste trop simplificateur) comme des produits hybrides. Dans le cas que nous étudions, ils sont un signe factice de la sinité.

Si Mulan a essuyé les critiques en Chine concernant son " manque d'authenticité » (et pour cause, nous l'avons vu), Disney avait mis certains moyens en ouvre, non pour faire un authentique dessin animé chinois, mais pour s'imprégner de ce pluralisme : voyages sur le terrain, recherches des codes de l'art chinois, style " graphique » confié au soin du dessinateur taïwanais, Chen Yi Chang. ${ }^{54}$ Rien n'y fit, car malheureusement pour Disney le film fut largement boudé dans les salles chinoises : Disney n'était pas assez authentiquement chinois pour les Chinois. 
De même, l'alliage entre ancien et nouveau (ou traditionnel et moderne) se décante dans des conditions similaires. Ce qui est «traditionnel »-la société orientale (la Chine)- n'envisage nullement sa modernité : qu'y a t-il en effet de moderne dans la Chine qui transparait à travers Mulan, Kungfu panda ? Certes rien, et la tradition agit comme un discours hypnotique, plonge les imaginaires dans la nostalgie de ce qui " a toujours été ", empêchant par là tout mouvement du monde moderne oriental : à chaque fois, ces films d'animation et le cortège de signes qui les entoure, perpétuent et approfondissent la coupure historique, imposant ce déni de l'évolution; ils contribuent en cela à la version atemporelle de l'histoire de la Chine, car la modernité est vécue comme une menace d'effacement de l'authenticité, (particulièrement vrai quand on en vient à parler de « l'Asie »), une perte de soi irréversible, un morceau d' « âme » déchiré. Cette impossibilité d'hybridité, la peur de la perte d'identité culturelle, aujourd'hui, est un rêve postmoderne partagé par les deux parties, et pousse dans le sens d'une survalorisation du passé. ${ }^{55}$ Et la Chine en intériorisant ce discours s'embourbe dans un passé fait sur mesure, mais qui lui échappe (le fantasme d'une histoire la « civilisation chinoise » qui remonte de plus en plus loin dans le temps, contribue à creuser un peu plus le gouffre temporel dans lequel elle se complait). ${ }^{6}$

71 De ce fait, la privation d'histoire renforce la conviction qu'il existe des essences. Animaux ou fossiles, voici renouvelée la vision immobile.

Dix ans après Mulan, on ne sait si le phénomène est comique ou tragique, mais Kungfu Panda, qui suit à la lettre la même recette sinisante que son aîné, retient toute l'attention en Chine. En tous les cas, son succès commercial sans précédent, comme l'appel au boycott du dessin animé sur certains sites internet chinois (pour différentes raisons, l'un d'entre eux dénonce le pillage de " l'héritage culturel chinois » par Hollywood, un autre est scandalisé par l'utilisation que fait Dreamworks de la figure du panda, alors que le Sichuan -zone d'habitat de l'animal- est tragiquement sinistré par le tremblement de terre) attestent de cette illusion globalisante. Le tour est joué, de pouvoir faire croire à un public chinois que la richesse culturelle de la Chine n'est faite que de pandas et de cris animaux remplis de " qi », doublé par sa star muette. L'assimilation du discours spectaculaire orientaliste à l'échelle du globe en est étonnante, tant de rapidité (à peine dix ans) que d'absurdité, c'est un retournement en bonne et due forme. Ziauddin Sardar avait déjà perçu cette tendance postmoderne par exemple, chez une partie de la jeunesse sud-est asiatique à se représenter au travers des critères MTV (Music Television) : « la différence c'est que maintenant, c'est l'Asie qui s'illusionne sur elle même ».57 L'aliénation de "l'oriental » est complète lorsque celui-ci recrée son propre manque. Il parle de lui, mais ne dit rien ; muet, fixe, incapable de s'imaginer autrement que dans la copie de l'Occident : il est à lui son simulacre.

\section{Conclusion}

La sinité animée en passe de devenir la Chine ; pour s'en convaincre, il suffit de jeter un œil sur la production cinématographique chinoise : de la pléthore de films historiques qui n'en finit plus de sommeiller dans la grandeur passée aux animations du studio de Shanghai (pourtant à une époque, le bastion du très créatif Te Wei) copiant, eux, la recette « occidentale » (images de synthèse, 
doublage des voix par les stars de la télévision ou du cinéma, etc.)..$^{8}$

Le marché du film est une Jiang Qing diffuse, qui impose sa tyrannie des modèles et déplace la Chine dans ses propres orients. Le dragon insouciant peut désormais mimer seul son orientalité. ${ }^{59}$

Bambi quant à lui s'ignorait hybride, et n'a donc pas (re)produit le mythe de la sinité. Saugrenu ? Pas le moins du monde : mais qui sait que Tyrus Wong, dessinateur d'origine chinoise, immortalisa le décor où gambade le faon, en puisant dans les rouleaux horizontaux de la peinture Song ? ?0 $^{60}$

\section{Notes}

1 Nous prenons pour objet deux dessins animés des studios d'animation Disney et Dreamworks, qui ont réalisé respectivement Mulan (1998) et Kungfu Panda (2008), deux animations «majeures » du point de vue commercial et critique, et qui situent l'action de leur récit en Chine.

2 Il s'agit donc du discours orientaliste mis à jour par Edward Said dans L'orientalisme : l'Orient créé par l'Occident, Paris, Seuil, 1980. Les continuateurs de la théorie postcoloniale comme Gayatri Spivak se sont attachés à ce type de " personnage secondaires » dans les romans, révélateurs d'une sorte d'inconscient de ce discours (voir Bart Moore-Gilbert, Postcolonial Theory : Contexts Practice and Politics, London, Verso, 1997). Les critiques postcoloniaux, tels par exemple Ziauddin Sardar ont, de leur côté, décelé cette tendance dans les médias de la culture populaire (films, télévision, etc.).

3 Roland Barthes, Mythologies, Paris, Seuil, 1957, pp.193-194.

4 Roland Barthes, Mythologies, Paris, Seuil, 1957.

5 Les lecteurs du mythe (comme du stéréotype) que nous sommes ne distinguent plus la superposition entre sens « premier » et sens mythologique.

6 Joseph M. Chan, «Disneyfying and Globalizing the Chinese Legend of Mulan », in Joseph Chan et Bryce Mc Intyre, In Search of Boundaries: Communication, Nation States and Cultural Identities, West Port, Albex publishing, 2002, p. 239. " Mulan is a nice story, but where are the great Chinese dragons and folklore and mythical creatures? When you think of China, one of the first things you think about are those hazy mountains and dragons everywhere. "

7 Gregory Lee a montré, à divers endroits, que les stéréotypes sur la Chine ont souvent tendu à être soit xénophobes (le chinois fumeur d'opium, Fu Manchu symbole du péril jaune menaçant le monde), soit ridicules (par exemple, le personnage de Hop Sing).

8 Sous de nombreuses formes (les croyances, la culture populaire, les récits légendaires, etc.), il existe un imaginaire dense, une richesse de références quant à ce bestiaire (par exemple, on recense au moins trois grandes catégories de dragons : célestes, aquatiques, terrestres, associé à des formes ou couleurs diverses.) Et il en va de même pour les autres (voir encore la symbolique du serpent analysée en détails par Kin-Chung Ho, « Le serpent blanc, figure de la liberté féminine », Association française d'études chinoises, Printemps 1992, volume XI, pp. 57-86.)

9 Roland Barthes, "La rhétorique de l'image » in Euvres complètes Tome II, Paris, Seuil, p. 573 .

10 Rappelons que Barthes prend ici pour exemple une image publicitaire de Panzani.

11 Le titre international est le même, sauf pour quelques pays, par exemple en Chine où le dessin animé est titré Gongfu Xiongmao 功夫熊猫 (d'ailleurs, un tel titre chinois peut paraître étonnant ; 功夫 renvoie certes à « kung-fu », mais ne traduit absolument pas la saturation martiale présente dans le terme « occidental »).

12 De tels mécanismes "d’inversion », de renversements des causes et effets dans les savoirs culturels, a été analysé bien plus finement qu'on ne le fait ici par Kojin Karatani, The Origins of Modern Japanese Literature, Durham and London, Duke 
University Press, 1993.

13 Barthes, « La rhétorique de l’image », p. 575

14 En soit, la scène se révèle être fidèle aux réalités guerrières auxquelles devait faire face l'empire. L'édifice qui ne répondait pas forcément à une symbolique d'étendue et de puissance de la Chine (l'endroit en question n'a été que très récemment réinvesti par un discours " romantique », lié au fantasme de grandeur de l'espace national chinois), symbolisait la vulnérabilité aux attaques. Dans le passé, la mémoire collective projette sur la muraille l'ombre de guerres sans fin et de parents ou proches tombés à son pied.

15 山水, littéralement " montagne et eau », désigne la peinture " traditionnelle » de paysage (voir par exemple Yolaine Escande, Montagne et eau : la culture du shanshui, Paris, Hermann, 2005.)

16 Roland Barthes, « La rhétorique de l'image », p. 586.

17 On pense par exemple aux films d'un Quentin Tarantino qu'on hésite toujours à classer entre un ridicule qui s'ignore ou un sens suprême de l'auto-dérision.

18 La chanson phare du film ne nous dit elle pas " Everybody wants to kungfu fighting »?

19 François Cheng, L'espace du rêve : mille ans de peinture chinoise, Paris, Phébus, 1981.

20 L'un des acteurs du doublage déclare significativement : " Le film se passe à un endroit particulier, il fallait en faire ressortir les particularités pour que le public se sente y être. Les réalisateurs ont utilisé la peinture chinoise de paysage et de construction pour donner au spectateur une impression inoubliable. J'avoue n'être jamais allé en Chine, mais si j'y vais je dirai sûrement : Oh! C'est comme les paysages de «Kung-fu panda »!» (voir « Kung-fu Panda », World Screen 世界電影, Hong-Kong, Juin 2008, $\mathrm{n}^{\circ}$ 474, p. 40).

21 Les animaux aux formes et attitudes humaines ont, dès les débuts, inspiré grandement le fondateur : il prendra pour modèle les meilleurs illustrateurs européens des 19 ème et début du 20ème siècles (par exemple, l'illustrateur allemand Heinrich Kley, à qui l'ont doit la fameuse « danse des éléphants » de Fantasia). L'illustration de Disney en retient tout l'aspect délicat et drôlatique, dont l'emblème de la souris anthropomorphique, Mickey Mouse, résume à elle seule tout l'héritage (voir Il était une fois Walt Disney : aux sources de l'art des studios Disney, Paris : Réunion des musées nationaux Montréal : Musée des beaux-arts de Montréal, 2007, p. 104. p. 6).

22 Joseph Chan, "Disneyfying and Globalizing the Chinese Legend of Mulan » in Joseph Chan et Bryce McIntyre (Dir.), In Search of Boundaries: Communication, Nation States and Cultural Identities, West Port, Albexpublishing, 2002, p. 231.

23 Voir par exemple, Jacques Pimpaneau, Anthologie de la littérature chinoise classique, Philipe Piquier, 2004, p. 242-244.

24 Barry Cook et Tony Bancroft, « Mulan », Walt Disney Pictures, 1998.

25 Barthes, Mythologies.

26 Chan, « Disneyfying and Globalizing the Chinese Legend of Mulan », p. 237.

27 Chan, « Disneyfying and Globalizing the Chinese Legend of Mulan », p. 235, " It was more acceptable to introduce animals sidekicks. »

28 Nous pourrions également consacrer toute une partie à la tradition des paysages disneyens, à ses caractéristiques. La recherche picturale qui accompagne l'élaboration d'un décor fait l'objet d'une grande attention, les dessinateurs de Disney puisent ainsi dans diverses ressources, synthétisant une multitude d'influences. La peinture régionaliste américaine, par exemple, donne à Disney l'idée d'ouvrir ses films par des plans panoramiques très larges, ce qui permet de conférer une impression de décor grandiose. Ainsi, la plupart des scènes d'ouverture des dessins animés Disney, donnent à voir un paysage dans son ensemble, une prise de vue par dessus qui découvre un panorama. Fait intéressant, « les arts orientaux » (les miniatures persanes, les estampes japonaises, peinture chinoise des Song) ont souvent fait l'objet d'un intérêt certain dans plusieurs animations (voir Il était une fois Walt Disney : aux sources de l'art des studios Disney, p. 214.)

29 A notre connaissance, aucune étude approfondie n'a été réalisée sur la « politique » -le politiquement non correct- des studios d'animation. 
30 Pour qui est familier d'un tel univers, on reconnaîtra par exemple Oscar, l'« anti-Nemo » de Rob Letterman, Vicky Jenson et Bibo Bergeron, " Gang de requins », Dreamworks Animation, 2004 ; le lion Alex dans Eric Darnell et Tom Mc Grath, « Madagascar », Dreamworks Animation, 2005 ; ou encore ce « siffleur professionnel », roi des lémuriens, King Julian. Toutes ces animations, à commencer par Shrek, entendent revisiter à leur manière le monde conventionnel des dessins animés et des contes de fées. Relevons dans ce contexte d'industrie culturelle, la critique idéologique de Slavoj Žižek sur le mécanisme de l'autodérision archétypale de la configuration de la « croyance » qui marque nos manières d'être et d'agir : en somme, personne n'y croit, certes, « l'antihéros » peut même se gausser de soi, cependant tout reste en l'état, et tout est fait pour que cela reste. En parlant de Kungfu Panda, Žižek nous dit : « Le film finit par prendre au sérieux la cible même de ses blagues incessantes ». (Slavoj Žižek, Après la tragédie, la farce !, Flammarion, Paris, 2010, p. 82). Et de fait, une telle critique peut être étendue à l'orientalisme sous-jacent du film : Kungfu Panda joue sur les bords du rire, pour que finalement, sa parodie « boucle la boucle » sur ses propres images de l'Orient ( "La surprise vient de ce que cette autodérision continuelle n'entrave aucunement l'efficacité du spiritualisme oriental ». Žižek, Après la tragédie, la farce !, p. 82).

31 Gregory Lee, « Mulan: Representation, Globalization and the Unforbidden City » in Modernités chinoises, Lyon, AEC-UJM Faculté des langues Université Jean Moulin-Lyon 3, 2004, p. 227.

32 Nous nous appuyons ici sur la continuité de l'analyse du discours orientaliste, effectuée notamment dans les travaux de Ziauddin Sardar, et plus spécifiquement sur la Chine, de Gregory Lee. Le sauvage, l'animal, le mystérieux, l'occulte, constellent l'imaginaire colonial ; a contrario, les autres parties du globe, les « métropoles », ne souffrent pas de ce type d'association. Pour rester dans le domaine de l'animation, « Ratatouille » des studios Pixar, par exemple, joue sur les concepts de francité, sans en donner cette tournure atemporelle. Le seul animal à cornes qui puisse encore provoquer l'imaginaire sur un pays « occidental » tel que la France, c'est l'escargot et la francité qui lui est attachée : mais la cuisine est-elle à la France, ce que le kung-fu est à la Chine?

33 En y accolant le sobriquet de " fast food du cinéma moderne », Ziauddin Sardar s'attaque entre autre au récit de Pocahontas (qui touche rappelons-le, au délicat sujet du sort des Indiens natifs d'Amérique du Nord) qu'il voit comme une représentation dans une galerie de représentations. Mulan s'il peut être réinscrit dans la lignée d'une parole américaine sur la sinité, ne traite pas aussi frontalement du problème par exemple des camps pour immigrés Chinois (Ziauddin Sardar, Postmodernism and the Other: the New Imperisalism of Western Culture, Chicago, London, Pluto Press, 1998, p. 98).

34 Ziauddin Sardar, Orientalism, p. 104, "For an exotic escapist entertainment. »

35 Gregory Lee, Chinas Unlimited: Making the Imagineries of China and Chineseness, Londres, Curzon Press, 2001, p. 13.

36 Lee, « Mulan: Representation, Globalization and the Unforbidden City », p. 228, « Mulan is made in an American historical context of a tradition of recuperation and reinvention of chineseness. »

37 Chan, « Disneyfying and Globalizing the Chinese Legend of Mulan », p. 228.

38 Selon Sheng-Mei Ma, l'anglicisation du nom de certains personnages, par exemple, permet une plus grande familiarité contextuelle pour le spectateur occidental (Sheng-Mei Ma, The Deathly Embrace: Orientalism and Asian American Identity, Minneapolis London, University of Minnesota Press, 2000, p. 126).

39 Gregory Lee, Chinas Unlimited, p. 11. Nous reprenons, en la modifiant pour notre propos, l'analyse de l'auteur d'un passage d'une nouvelle de Duo Duo, « Hui Jia » 回家. Lorsque le personnage principal qui vit en Angleterre se trouve devant une peinture à l'huile représentant la Chine, G. Lee note : " chineseness made present by a sense of Chinese absence, is represented no only by being elsewhere, being in England, and by English reactions to the Chinese presence, but also by the traces of chineseness, by the appearance of chineseness, by chinese presences that fail to transform into Chinese valence for the narrator. »

40 On pense au concept de " mimicry » élaboré par Homi Bhabha (voir Mrinalini Greedharry, Postcolonial Theory and Psychoanalysis, New-York, Palgrave Mcmillan, 2008, pp.87-88) : aucun hasard à ce que Jackie Chan "double » un personnage « Singe » : les libres associations en termes d'imitation sont ici transparentes... 
41 Témoin a contrario, la désaffection médiatique du film du réalisateur Jia Zhangke, en lice au même moment, 24 city sur les difficultés urbaines de ce siècle en Chine Populaire. Quelques critiques éparses remarquent le film, mais assurément l'enthousiasme soulevé n'est d'aucune commune mesure avec l'animation hollywoodienne. Prestige du spectacle, qui vient confirmer cette préférence pour le fantasme orientaliste.

42 Guy Debord, Commentaires sur la société du spectacle, Paris, Gallimard, 1988, pp. 63-64.

43 Lee, « Mulan: Representation, Globalization and the Unforbidden City », p. 234.

44 Debord, Commentaires sur la société du spectacle, p. 24.

45 Debord, Commentaires sur la société du spectacle, p. 24.

46 Ziauddin Sardar, Postmodernism and the Other.

47 Barthes, Mythologies, p. 227.

48 Sardar, Postmodernism and the Other, p. 86.

49 Et le fait que Po incarne la figure de l'antihéros n'y change rien, au contraire. Souvenons-nous de la critique de l'autodérision, et à nouveau citons Žižek : " En raison de son caractère ubiquitaire, l'idéologie peut apparaître sous sa forme contraire, comme non-idéologie, comme le noyau de notre identité humaine, une fois celle-ci dépouillée de tous ses oripeaux idéologiques. » (Žižek, Après la tragédie, la farce !, p. 64).

50 Chan, « Disneyfying and Globalizing the Chinese Legend of Mulan », pp. 240-241.

51 Sardar, Postmodernism and the Other, p. 28. "When non western cultural artefacts appear in the West they do so striclty as ethnic chic or empty symbols. »

52 Sardar, Postmodernism and the Other, p. 90.

53 Chan, " Disneyfying and Globalizing the Chinese Legend of Mulan », p. 228. « Transculturation is a form of cultural borrowing in which one culture reconfigures another culture for its own purpose. »

54 Chan, « Disneyfying and Globalizing the Chinese Legend of Mulan », pp. 234-235.

55 Gregory B. Lee et Sunny K. Lam, « Wicked Cities: The Other in Hong Kong Science Fiction » in Modernités chinoises, Lyon, AEC-UJM Faculté des langues Université Jean Moulin-Lyon 3, 2004.

56 Jean-François Billeter, Chine trois fois muette, Paris, Allia, 2006, p. 75.

57 Sardar, Postmodernism and the Other, p. 136.

58 Te Wei et son équipe, des années 1950 aux années 1980 entrecoupées de troubles politiques, ont réalisé des monuments de l'animation, en parachevant une technique qui fusionnait peinture et animation. Ils réussirent notamment à animer les peintures de Qi Baishi.

59 En quelque sorte, une version en négatif de la « mimicry » dont parle Homi Bhabha, une inversion du narcissisme occidental (voir Moore-Gilbert, Postcolonial Theory : Contexts Practice and Politics, p. 132).

6o Il était une fois Walt Disney : aux sources de l'art des studios Disney, Paris : Réunion des musées nationaux Montréal : Musée des beaux-arts de Montréal, 2007, p. 214.

\section{Pour citer cet article}

Référence électronique

Pierre-Mong LIM , « Le panda hypocrite : sinité, studios d'animation et discours orientaliste postmoderne », Transtext(e)s Transcultures 跨文本跨文化 [En ligne] , 6 | 2011 , mis en ligne le 07 avril 2011, Consulté le 26 avril 2011. URL :

http://transtexts.revues.org/index408.html

\section{Auteur}


Pierre-Mong LIM

Pierre-Mong LIM est doctorant à I'Université Lyon 3, rattaché à l'Institut d'Etudes

Transtextuelles et Transculturelles.

\section{Droits d'auteur}

(C) Tous droits réservés 